\title{
A data-driven method to predict achievability of clinical objectives in IMRT
}

Vaitheeswaran Ranganathan ( $\nabla$ vaitheeswaran.r@philips.com )

Philips (India)

\section{Research Article}

Keywords: Optimization, IMRT, objective function, radiotherapy, Auto Plan, inverse planning

Posted Date: July 19th, 2021

DOl: https://doi.org/10.21203/rs.3.rs-731148/v1

License: (c) (i) This work is licensed under a Creative Commons Attribution 4.0 International License. Read Full License 


\section{A data-driven method to predict achievability of clinical objectives in IMRT}

\section{Vaitheeswaran Ranganathan, Philips Healthcare, Bangalore, India}

\section{Abstract:}

5 When specifying a clinical objective for a target volume and normal organs/tissues in IMRT planning, the user may not be sure if the defined clinical objective could be achieved by the optimizer. To this end, we propose a novel method to predict the achievability of clinical objectives upfront before invoking the optimization. A new metric called "Geometric Complexity (GC)" is used to estimate the achievability of

10 clinical objectives. Essentially GC is the measure of the number of "unmodulated" beamlets or rays that intersect the Region-of-interest (ROI) and the target volume. We first compute the geometric complexity ratio (GCratio) between the GC of a ROI in a reference plan and the GC of the same ROI in a given plan. The GCratio of a ROI indicates the relative geometric complexity of the ROI as compared to the same ROI in

15 the reference plan. Hence GCratio can be used to predict if a defined clinical objective associated with the ROI can be met by the optimizer for a given case. We have evaluated the proposed method on six Head and Neck cases using Pinnacle3 (version 9.10.0) Treatment Planning System (TPS). Out of total of 42 clinical objectives from six cases accounted in the study, 37 were in agreement with the prediction, which implies an

20 agreement of about $88 \%$ between predicted and obtained results. The results indicate the feasibility of using the proposed method in head and neck cases for predicting the achievability of clinical objectives. 


\section{A data-driven method to predict achievability of clinical objectives in}

\section{IMRT}

\section{INTRODUCTION}

Intensity modulated radiation therapy (IMRT) has grown as an effective way of producing a conformal dose to tumor, while effectively sparing the surrounding normal

30 tissues and organs. The main goal of optimization in IMRT is to find parameters that will yield the best possible treatment plans under given clinical and technical conditions. In the current practice of IMRT planning, one of the common approaches is that a user would first define a set of initial clinical objectives for target volumes (E.g. Minimum PTV Dose of $6300 \mathrm{cGy}$ ) and perform the optimization. After achieving the target volume

35 objectives, user would start including Organs-at-risk (OAR) objectives (E.g. spinal cord Maximum Dose of 4500 cGy) one by one and perform several re-optimizations. From here on, the process gets more complicated and the user is required to carefully tweak the objective parameters in order to strike a balance between target coverage, target homogeneity and OAR sparing [1]. This generally involves several optimizations to

40 arrive at an optimal objective setting. Often, for difficult treatment plans, the user may not be sure if the defined clinical objective could be achieved by the optimizer. In many situations, the defined clinical objective goes unachieved by the optimizer. But this realization happens only after performing one or many optimizations. This leads to several backtracking steps and hence the process becomes ineffective and time

45 consuming. Moreover, due to these difficulties, the resulting plan quality becomes highly dependent on the ability of the treatment planners to meet the specified objectives [2]. 
Researchers have investigated algorithmic methods to make IMRT planning more efficient and less dependent on the expertise of the treatment planners [3-9]. Some

50 researchers have explored data-driven approaches as well with the same research intent [10-13]. Such data-driven methods have been proven successful for predicting achievable dose levels for clinical objectives before invoking the actual optimization.

We propose a novel data-driven method to improve the efficiency of IMRT optimization

55 process. Our method allows predicting the achievability of clinical objectives upfront before invoking the optimization, thereby eliminating the need for several trials and errors in fine tuning the objective parameters. This study evaluates the feasibility of the proposed method on Head and Neck cases.

60 MATERIALS AND METHODS:

Geometric Complexity (GC):

Geometric complexity (GC) is the measure of the number of "unmodulated" beamlets or rays that intersect the Region-of-interest (ROI) and the composite target volume (i.e. the volume containing all target volumes). The GC computed for a ROI is given by

65

$$
G C=\frac{n+1}{N_{T} / V}=\frac{n+1}{N}
$$

Where, $\mathrm{n}$ is the number of beamlets that pass through the ROI and target volume for a

70 given plan, $V$ is the volume of the target volume (e.g. the volume of the tumor to be 
irradiated), $\mathrm{N}_{\mathrm{T}}$ is the total number of beamlets passing through the target volume, and $N=N_{T} / V$ is the number of available beamlets per unit volume of the target.

Figures 1 diagrammatically illustrates two examples of a target volume and an OAR

75 being irradiated by a single radiation beam. In this example, the number $N_{T}$ of available beamlets is four. (This is diagrammatic: in practical IMRT cases $N_{T}$ is typically on the order of hundreds or thousands). In the example of Figure 1a, all four available beamlets that intersect the target volume also intersect the OAR, so that $n=4$. This results in a high value for the GC metric of Equation (1), indicating high geometrical complexity and 80 a low likelihood of achieving the objectives associated with the OAR after optimization.

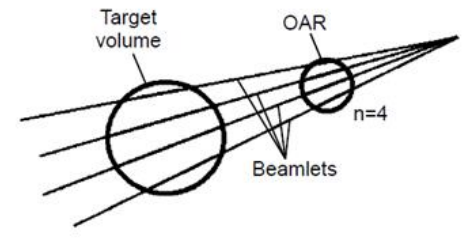

(1a)

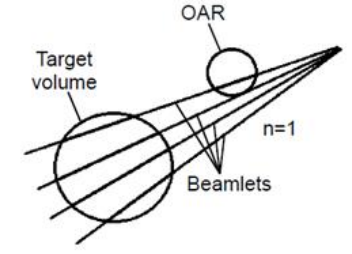

(1b)

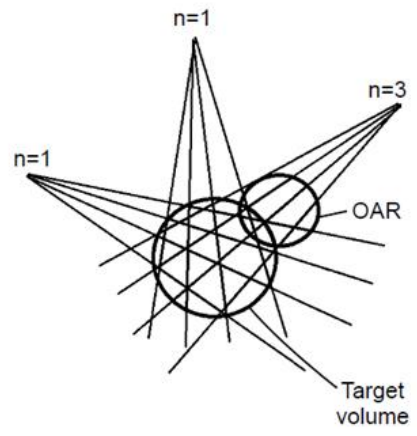

(1c)

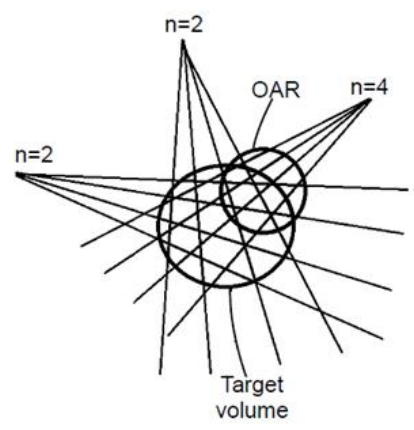

(1d)

Figure 1: Diagrammatically illustrated examples (1a-1d) of geometric complexity estimation based on the position of OAR with respect to target volume $(1 a-1 b)$ and the level of target-OAR overlap $(1 c-1 d)$ 
95 By comparison, Figure 1b shows a different IMRT geometry in which only one of the beamlets that intersect the target volume also intersects the OAR, leading to $n=1$. This results in a low value for the GC metric of Equation (1), indicating low geometrical complexity and a high likelihood of achieving the objectives associated with the OAR after optimization. (For example, that single beamlet intersecting the OAR could be 100 shuttered off by the optimized MLC configuration).

In another example illustrated in Figures 1c and 1d, each radiation beam is modulated into four beamlets, so that for the three radiation beams $N_{T}=4 \times 3=12$. In the example of Figure 1c, one beamlet of the leftmost radiation beam also intersects the

105 OAR, one beamlet of the middle radiation beam also intersects the OAR, and three beamlets of the rightmost radiation beam also intersect the OAR. Thus, $n=1+1+3=5$, and from Equation (1) the GC metric is $\left(\frac{6}{12}\right) V$. In the example of Figure 1d, two beamlets of the leftmost radiation beam also intersects the OAR, two beamlets of the middle radiation beam also intersects the OAR, and all four beamlets of 110 the rightmost radiation beam also intersect the OAR. Thus, $n=2+2+4=8$, and from Equation (1) the GC metric is $\left(\frac{9}{12}\right) V$. It follows that the IMRT geometry of Figure 1c is more likely to yield an achievable IMRT plan optimization (because of its lower GC) as compared with the IMRT geometry of Figure 1d (because of its higher GC). 


\section{Geometric complexity ratio}

We first create a reference plan in which all the clinical objectives have been met through the optimization process. The GC associated with each segmented clinical structure or

120 Region of Interest (ROI) is computed for the reference plan. The GC obtained per ROI for the reference plan is considered as the base or reference value i.e. $\mathrm{GC}_{\text {ref. }}$.

$$
G C_{\text {ref }}=\frac{n_{\text {ref }}+1}{N_{T_{v} \text { ref }} / V_{\text {ref }}}=\frac{n_{\text {ref }}+1}{N_{\text {ref }}}
$$

Where, $n_{\text {ref }}$ is the number of beamlets that pass through the ROI and target volume for a reference plan, $V_{\text {ref }}$ is the volume of the target volume (e.g. the volume of the tumor to be irradiated), $\mathrm{N}_{\mathrm{T}}$, ref is the total number of beamlets passing through the target volume in

130 the reference plan and $\mathrm{N}_{\text {ref }}=\mathrm{N}_{\mathrm{T} \text {,ref }} / \mathrm{V}_{\text {ref }}$ is the number of available beamlets per unit volume of the target volume in the reference plan.

Dividing equation 1 by equation 2 , we get geometric complexity ratio $\left(\mathrm{GC}_{\text {ratio }}\right)$ of a $\mathrm{ROI}$ as shown below.

$$
G C_{\text {ratio }}=\frac{\frac{n+1}{N}}{\frac{n_{r e f}+1}{N_{\text {ref }}}}
$$

140 It is to be note that if the ROI is a target volume, then ' $n$ ' refers to the number of beamlets that intersect target volume and all other ROIs getting included in the optimization. 


\section{Predicting the achievability of clinical objectives}

Essentially our approach involves the comparison of geometric complexity of a given plan to that of a reference plan to estimate the achievability of clinical objectives. The $\mathrm{GC}_{\text {ratio }}$ of a $\mathrm{ROI}$ indicates the relative geometric complexity of the ROI as compared to the same ROI in the reference plan. Achievability of a clinical objective associated with

150 the ROI can be perceived as the inverse of $\mathrm{GC}_{\text {ratio }}$ of the ROI. Hence $\mathrm{GC}_{\text {ratio }}$ can be used to predict if a defined clinical objective associated with the ROI can be met by the optimizer for a given case. Basically a higher $\mathrm{GC}_{\text {ratio }}$ indicates a lesser likelihood for the optimizer to achieve the clinical objective defined for a given ROI. Similarly, a lower $\mathrm{GC}_{\text {ratio }}$ indicates a higher likelihood for the optimizer to achieve the clinical objective 155 defined for the given ROI.

In the examples illustrated in Figures 1a to 1d, assume that 1a and 1c are reference plans and $1 \mathrm{~b}$ and $1 \mathrm{~d}$ are given or current plans. Also assume that the OAR objectives in the reference plans 1a and 1c have been met by the optimizer. The estimated $\mathrm{GC}_{\text {ratio }}$ for OAR

160 in plan $1 \mathrm{~b}$ with respect to plan $1 \mathrm{a}$ is 0.25 , which implies that if a planner defines same objective setting for the OAR in current plan, it is highly likely that the objective will be met by the optimizer. Similarly, the estimated $\mathrm{GC}_{\text {ratio }}$ for $\mathrm{OAR}$ in plan $1 \mathrm{~d}$ with respect to plan 1c is 1.5 , which implies that if a planners defines same objective setting for the OAR in current plan, it is highly likely that the objective will not be met by the optimizer.

However, these numerical values may not be intuitive for the user. Hence, we have interpreted the achievability of the objectives (i.e. likelihood of achieving an objective) 
based on the $\mathrm{GC}_{\text {ratio }}$ value using different schemes as given in Table I. The basic assumption when predicting the achievability of a given objective is that the objective

170 parameters (i.e. dose, volume and weight) associated with that objective is same as that used in the reference plan. It is to be noted that the clinical objectives in the reference plan(s) must have been achieved in order to make accurate predictions. Figure 2 shows the flowchart of the proposed method.

175

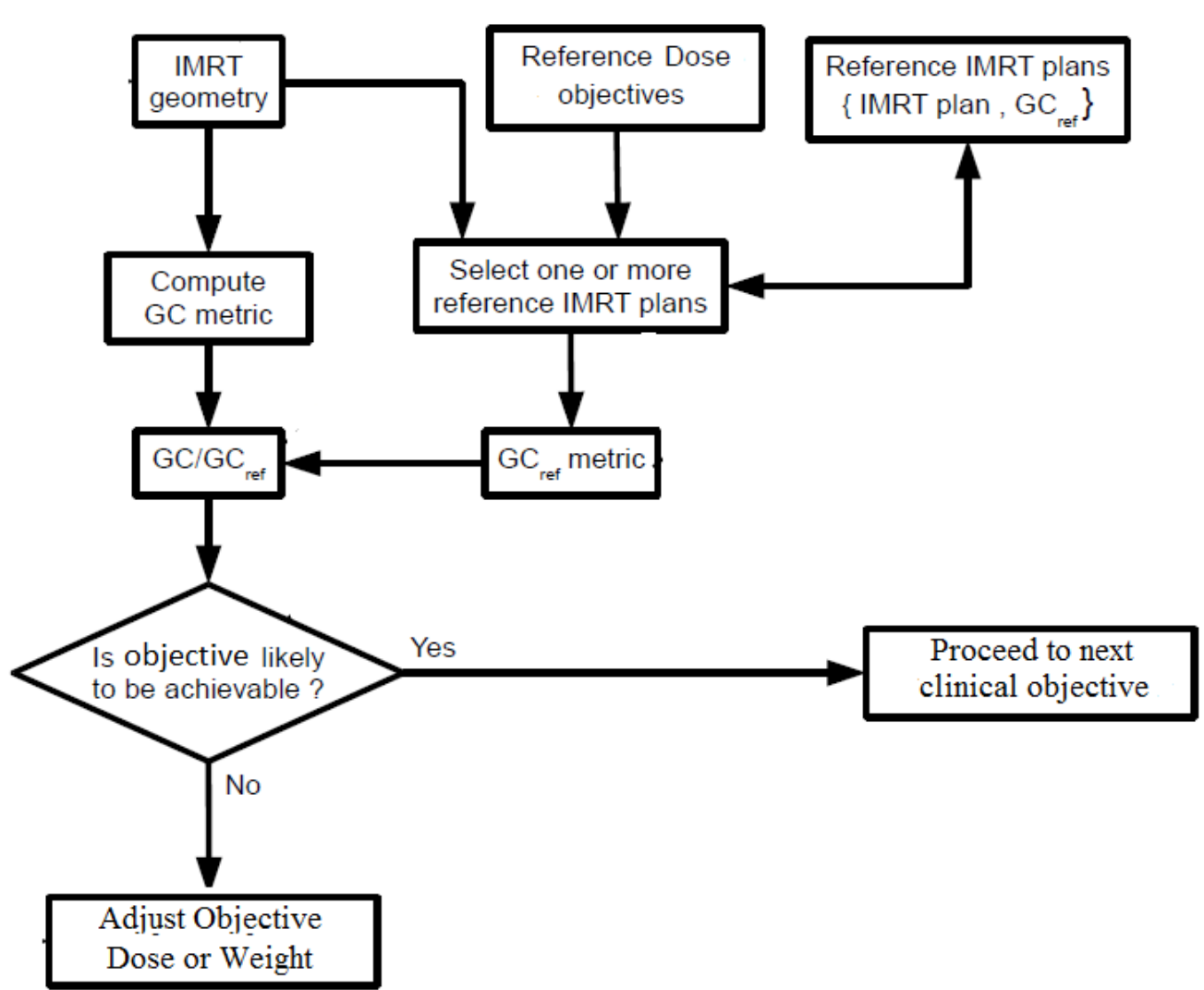

Figure 2: Flowchart of the proposed method for predicting the achievability of clinical objectives 


\section{Study methodology}

Pinnacle $^{3}$ (version 9.10.0) Treatment Planning System (TPS) was used for the whole study for contouring, dose computation and optimization. The optimization was done using DMPO module in which a minimum segment MU of 4 and minimum segment area

195 of $4 \mathrm{~cm}^{2}$ was used throughout the study. Varian Tx HDMLC machine was used for simulation purposes. The dose grid resolution was set to $0.3 \mathrm{~cm}$ in $\mathrm{X}, \mathrm{Y}$ and $\mathrm{Z}$ planes for dose computation.

First, a reference head and neck case with 7 beams was selected. For the reference plan, a

200 manual planning in a conventional manner was performed by adding clinical objectives one-by-one (in the order of target objectives, cord and brainstem objectives and parotid objectives) and tweaking the objective parameters over many optimizations (as described in the introduction) and reached an acceptable plan, wherein all defined objectives were met. The $\mathrm{GC}_{\text {ref }}$ values for each $\mathrm{ROI}$ included in the reference plan was computed. Six

205 head and neck cases of varying complexity was selected for the study. The beams angles were manually selected for all the cases. The GC values were computed for target volumes, spinal cord, parotids and brainstem in these plans. Our objective is to predict the achievability of clinical objectives associated with these ROIs by making use of the reference $\mathrm{GC}_{\text {ref }}$ values obtained in the reference plan. Equation 3 was used to compute

$210 \mathrm{GC}_{\text {ratio }}$ for all ROIs (including target volumes). The schemes listed in Table I were used to interpret the $\mathrm{GC}_{\text {ratio }}$ values. Different schemes were used for interpretation because at the time of study we were not sure which one will be suitable with respect to the selected reference plan. Our intention is to find an interpretation scheme that gives best agreement 
Table I: Interpretation of the achievability of the objectives (i.e. likelihood of achieving 215 an objective) based on the $\mathrm{GC}_{\text {ratio }}$ value using different schemes. Here $\mathrm{A}$ refers to Achievable, PA refers to possibly achievable, PNA refers to possibly not achievable and NA refers to not achievable.

220

\begin{tabular}{|c|c|}
\hline Scheme \# & Scheme \\
\hline 1 & $\begin{array}{c}\mathrm{GC}_{\text {ratio }} \leq 1=\mathrm{A} \\
1<\mathrm{GC}_{\text {ratio }} \leq 1.1=\mathrm{PA} \\
1.1<\mathrm{GC}_{\text {ratio }} \leq 1.2=\mathrm{PNA} \\
\mathrm{GC}_{\text {ratio }}>1.2=\mathrm{NA}\end{array}$ \\
\hline 2 & $\begin{array}{c}\mathrm{GC}_{\text {ratio }} \leq 1.15=\mathrm{A} \\
1.15<\mathrm{GC}_{\text {ratio }} \leq 1.2=\mathrm{PA} \\
1.2<\mathrm{GC}_{\text {ratio }} \leq 1.25=\mathrm{PNA} \\
\mathrm{GC}_{\text {ratio }}>1.25=\mathrm{NA}\end{array}$ \\
\hline 3 & $\begin{array}{c}\mathrm{GC}_{\text {ratio }} \leq 1.2=\mathrm{A} \\
1.2<\mathrm{GC}_{\text {ratio }} \leq 1.3=\mathrm{PA} \\
1.3<\mathrm{GC}_{\text {ratio }} \leq 1.35=\mathrm{PNA} \\
\mathrm{GC}_{\text {ratio }}>1.35=\mathrm{NA}\end{array}$ \\
\hline 4 & $\begin{array}{c}\mathrm{GC}_{\text {ratio }} \leq 1.2=\mathrm{A} \\
1.2<\mathrm{GC}_{\text {ratio }} \leq 1.35=\mathrm{PA} \\
1.35<\mathrm{GC}_{\text {ratio }} \leq 1.45=\mathrm{PNA} \\
\mathrm{GC}_{\text {ratio }}>1.45=\mathrm{NA}\end{array}$ \\
\hline
\end{tabular}

between predicted and obtained results with respect to the selected reference plan. Basically the achievability is interpreted as "Achievable (A)", "Possibly Achievable 
(PA)", "Possibly Not Achievable (PNA)" and "Not Achievable (NA)" based on GC ratio 240 values. After making the predictions with four different schemes, we included the clinical objectives one-by-one in the same order they were added in reference plan and performed optimization (without changing objective parameters) to get the actual results for each clinical objective. The same was repeated for all six cases.

\section{RESULTS:}

250

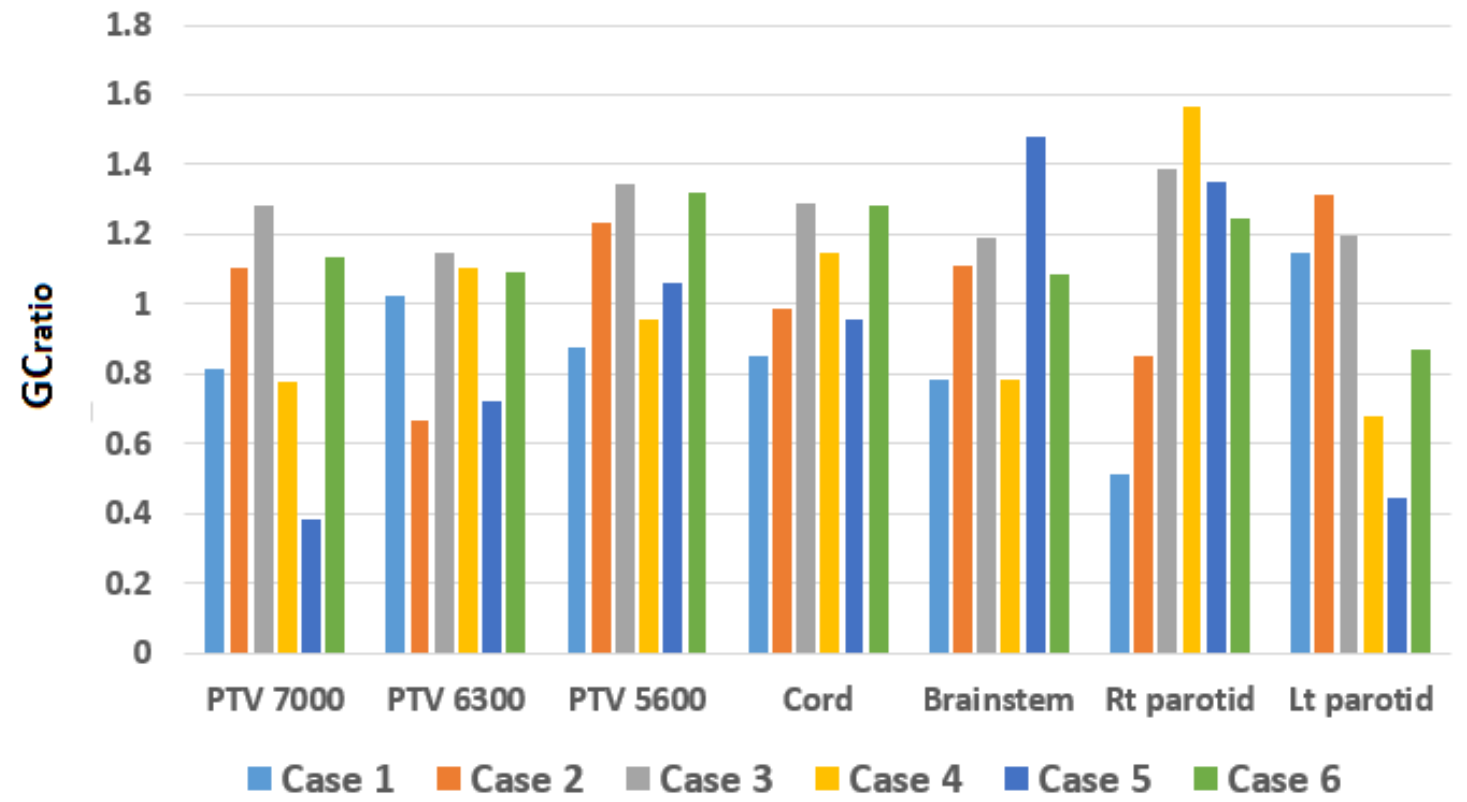

Figure 3: The variations in the geometric complexity for different ROIs in the six cases included in the study.

Table II: Comparison of $\mathrm{GC}_{\text {ratio }}$ values, estimated achievability using different schemes and obtained (actual) results after optimization for six head and neck cases. Here 'Ach' refers to Achieved and 'Not Ach' refers to Not Achieved. 
Table IIa: Head \& Neck - Reference case

\begin{tabular}{cccccccccc}
\hline \multirow{2}{*}{ Organ } & Objective & Dose & Volume & & Scheme & Scheme & Scheme & Scheme & Obtained \\
& Type & $(\mathbf{c G y})$ & $\mathbf{( \% )}$ & & $\mathbf{1}$ & $\mathbf{2}$ & $\mathbf{3}$ & $\mathbf{4}$ & Result \\
\hline PTV 7000 & Target Dose & 7000 & 95 & 1 & -- & -- & -- & -- & Ach \\
PTV 6300 & Target Dose & 6300 & 95 & 1 & -- & -- & -- & -- & Ach \\
PTV 5600 & Target Dose & 5600 & 95 & 1 & -- & -- & -- & -- & Ach \\
Cord & Max Dose & 4500 & 0 & 1 & -- & -- & -- & -- & Ach \\
B.Stem & Max Dose & 5400 & 0 & 1 & -- & -- & -- & -- & Ach \\
Parotid Rt & Mean Dose & 2600 & -- & 1 & -- & -- & -- & -- & Ach \\
Parotid Lt & Mean Dose & 2600 & -- & 1 & -- & -- & -- & -- & Ach \\
\hline \hline
\end{tabular}

Table IIb: Head \& Neck -Case 1

\begin{tabular}{cccccccccc}
\hline Organ & Objective & Dose & Volume & Scheme & Scheme & Scheme & Scheme & Obtained \\
& Type & $(\mathbf{c G y})$ & $(\boldsymbol{\%})$ & & $\mathbf{1}$ & $\mathbf{2}$ & $\mathbf{3}$ & $\mathbf{4}$ & Result \\
\hline PTV 7000 & Target Dose & 7000 & 95 & 1.104 & PA & A & A & A & Ach \\
PTV 6300 & Target Dose & 6300 & 95 & 0.667 & A & A & A & A & Ach \\
PTV 5600 & Target Dose & 5600 & 95 & 1.231 & NA & PNA & A & A & Ach \\
Cord & Max Dose & 4500 & 0 & 1.112 & PA & A & A & A & Ach \\
B.Stem & Max Dose & 5400 & 0 & 0.989 & A & A & A & A & Ach \\
Parotid Rt & Mean Dose & 2600 & -- & 0.854 & A & A & A & A & Ach \\
Parotid Lt & Mean Dose & 2600 & -- & 1.313 & NA & NA & PNA & PNA & Not Ach \\
\hline \hline
\end{tabular}


265 Table IIc: Head \& Neck - Case 2

\begin{tabular}{cccccccccc}
\hline \multirow{2}{*}{ Organ } & Objective & Dose & Volume & & Scheme & Scheme & Scheme & Scheme & Obtained \\
& Type & (cGy) & $(\boldsymbol{\%})$ & & $\mathbf{1}$ & $\mathbf{2}$ & $\mathbf{3}$ & $\mathbf{4}$ & Result \\
\hline PTV 7000 & Target Dose & 7000 & 95 & 0.811 & $\mathrm{~A}$ & $\mathrm{~A}$ & $\mathrm{~A}$ & $\mathrm{~A}$ & Ach \\
PTV 6300 & Target Dose & 6300 & 95 & 1.024 & PA & A & A & A & Ach \\
PTV 5600 & Target Dose & 5600 & 95 & 0.877 & A & A & A & A & Ach \\
Cord & Max Dose & 4500 & 0 & 0.852 & A & A & A & A & Ach \\
B.Stem & Max Dose & 5400 & 0 & 0.781 & A & A & A & A & Ach \\
Parotid Rt & Mean Dose & 2600 & -- & 0.514 & A & A & A & A & Ach \\
Parotid Lt & Mean Dose & 2600 & -- & 1.144 & PA & A & A & A & Ach \\
\hline \hline
\end{tabular}

Table IId: Head \& Neck - Case 3

\begin{tabular}{cccccccccc}
\hline \hline \multirow{2}{*}{ Organ } & Objective & Dose & Volume & & Scheme & Scheme & Scheme & Scheme & Obtained \\
& Type & $(\mathbf{c G y})$ & $(\boldsymbol{\%})$ & & $\mathbf{1}$ & $\mathbf{2}$ & $\mathbf{3}$ & $\mathbf{4}$ & Result \\
\hline PTV 7000 & Target Dose & 7000 & 95 & 1.285 & NA & NA & PA & PA & Not Ach \\
PTV 6300 & Target Dose & 6300 & 95 & 1.145 & PA & A & A & A & Not Ach \\
PTV 5600 & Target Dose & 5600 & 95 & 1.341 & NA & NA & PNA & PNA & Not Ach \\
Cord & Max Dose & 4500 & 0 & 1.291 & NA & NA & PNA & PNA & Not Ach \\
B.Stem & Max Dose & 5400 & 0 & 1.188 & NA & PA & A & A & Not Ach \\
Parotid Rt & Mean Dose & 2600 & -- & 1.385 & NA & NA & NA & PNA & Not Ach \\
Parotid Lt & Mean Dose & 2600 & -- & 1.193 & PNA & PA & A & A & Ach \\
\hline \hline
\end{tabular}


Table IIe: Head \& Neck - Case 4

\begin{tabular}{cccccccccc}
\hline \hline \multirow{2}{*}{ Organ } & Objective & Dose & Volume & Scheme & Scheme & Scheme & Scheme & Obtained \\
& Type & $(\mathbf{c G y})$ & $\mathbf{( \% )}$ & & $\mathbf{1}$ & $\mathbf{2}$ & $\mathbf{3}$ & $\mathbf{4}$ & Result \\
\hline PTV 7000 & Target Dose & 7000 & 95 & 0.775 & A & A & A & A & Ach \\
PTV 6300 & Target Dose & 6300 & 95 & 1.105 & PA & A & A & A & Ach \\
PTV 5600 & Target Dose & 5600 & 95 & 0.953 & A & A & A & A & Ach \\
Cord & Max Dose & 4500 & 0 & 1.145 & PA & A & A & A & Ach \\
B.Stem & Max Dose & 5400 & 0 & 0.782 & A & A & A & A & Ach \\
Parotid Rt & Mean Dose & 2600 & -- & 1.564 & NA & NA & NA & NA & Ach \\
Parotid Lt & Mean Dose & 2600 & -- & 0.678 & A & A & A & A & Ach \\
\hline \hline
\end{tabular}

270 Table IIf: Head \& Neck - Case 5

\begin{tabular}{|c|c|c|c|c|c|c|c|c|c|}
\hline Organ & $\begin{array}{c}\text { Objective } \\
\text { Type }\end{array}$ & $\begin{array}{l}\text { Dose } \\
\text { (cGy) }\end{array}$ & $\begin{array}{c}\text { Volume } \\
(\%)\end{array}$ & $\mathrm{GC}_{\text {ratio }}$ & $\begin{array}{c}\text { Scheme } \\
1\end{array}$ & $\begin{array}{c}\text { Scheme } \\
2\end{array}$ & $\begin{array}{c}\text { Scheme } \\
3\end{array}$ & $\begin{array}{c}\text { Scheme } \\
4\end{array}$ & $\begin{array}{c}\text { Obtained } \\
\text { Result }\end{array}$ \\
\hline PTV 7000 & Target Dose & 7000 & 95 & 0.385 & A & A & A & A & Ach \\
\hline PTV 6300 & Target Dose & 6300 & 95 & 0.724 & A & A & A & A & Ach \\
\hline PTV 5600 & Target Dose & 5600 & 95 & 1.061 & PA & A & A & NA & Ach \\
\hline Cord & Max Dose & 4500 & 0 & 0.958 & A & A & A & A & Ach \\
\hline B.Stem & Max Dose & 5400 & 0 & 1.481 & NA & NA & NA & NA & Not Ach \\
\hline Parotid Rt & Mean Dose & 2600 & -- & 1.349 & NA & NA & PNA & PNA & Not Ach \\
\hline Parotid Lt & Mean Dose & 2600 & -- & 0.446 & A & A & A & A & Ach \\
\hline
\end{tabular}


Table IIg: Head \& Neck - Case 6

\begin{tabular}{cccccccccc}
\hline Organ & Objective & Dose & Volume & & Scheme & Scheme & Scheme & Scheme & Obtained \\
& Type & (cGy) & $(\boldsymbol{\%})$ & & $\mathbf{1}$ & $\mathbf{2}$ & $\mathbf{3}$ & $\mathbf{4}$ & Result \\
\hline \hline PTV 7000 & Target Dose & 7000 & 95 & 1.135 & PA & A & A & A & Ach \\
PTV 6300 & Target Dose & 6300 & 95 & 1.094 & PA & A & A & A & Ach \\
PTV 5600 & Target Dose & 5600 & 95 & 1.317 & NA & NA & PNA & PNA & Not Ach \\
Cord & Max Dose & 4500 & 0 & 1.282 & NA & NA & PA & PA & Not Ach \\
B.Stem & Max Dose & 5400 & 0 & 1.088 & PA & A & A & A & Ach \\
Parotid Rt & Mean Dose & 2600 & -- & 1.244 & NA & PNA & PA & PA & Ach \\
Parotid Lt & Mean Dose & 2600 & -- & 0.872 & A & A & A & A & Ach \\
\hline \hline
\end{tabular}

Table II gives the comparison between predicted and obtained results for the four interpretation schemes. Figure $\mathbf{3}$ shows the geometric complexity ratio for different ROIs in the six cases included in the study. The cumulative $\mathrm{GC}_{\text {ratio }}$ values (i.e. sum of all $\mathrm{GC}_{\text {ratio }}$ values greater than 1) for Case 1 to Case 6 are 4.8, 2.2, 8.8, 4.5, 3.9, and 7.2 respectively.

280 We analyzed the results using Pearson correlation method. In order to perform correlation tests, a value of 1 was assigned to 'A', 0.5 to 'PA', 0.5 to 'PNA' and 0 to 'NA'. Also a value of 1 was assigned to the obtained result if the objective was met and a value of 0 if the objective was not met. Though the prediction using all four schemes have a positive correlation with the obtained results, scheme \# 2 has the maximum positive correlation 
285 (correlation was 0.81 with $\mathrm{r}^{2}$ value of 0.66 and $\mathrm{p}<0.005$ ) with the obtained results. It is evident from Table II that, out of the total of 42 clinical objectives from all six cases, 37 were in agreement with the prediction for scheme \# 2 which implies an agreement of about $88 \%$ between predicted and obtained results. Table III lists the percentage correlation and strength of correlation between predicted and obtained results for all

290 interpretation schemes. Please note that we have previously presented these results in AAPM 2016 annual conference [14] and also published as Patent [15].

\section{DISCUSSION}

The results indicate that it is feasible to use the proposed method to predict the 295 achievability of clinical objectives before invoking optimization. Except for Case 3, the prediction was accurate for other cases. For Case 3, the cumulative $\mathrm{GC}_{\text {ratio }}$ score is significantly higher, which indicates that the geometry of Case 3 is considerably different from that of reference plan. Basically a poor selection of reference plan can lead to inaccurate predictions. Hence, it is recommended to choose an appropriate reference plan

300 with respect to a given case to make accurate predictions. In practice, one can run a search on a database of previously optimized plans with estimated $\mathrm{GC}_{\text {ratio }}$ values for different ROIs and preferably select a plan for which the cumulative score is less than or equal to the number of ROIs (including target volumes) included in the optimization. The plan selected hereby can be used as a reference plan for making the predictions on 305 clinical objectives. This process is illustrated in Figure 4. 
Table III: The percentage agreement and strength of correlation between predicted and 310 obtained results for different interpretation schemes.

\begin{tabular}{|c|c|c|c|c|}
\hline Scheme \# & Scheme & \% agreement & Correlation & $\mathbf{r}^{2}$ \\
\hline 1 & $\begin{array}{c}\mathrm{GC}_{\text {ratio }} \leq 1=\mathrm{A} \\
1<\mathrm{GC}_{\text {ratio }} \leq 1.1=\mathrm{PA} \\
1.1<\mathrm{GC}_{\text {ratio }} \leq 1.2=\mathrm{PNA} \\
\mathrm{GC}_{\text {ratio }}>1.2=\mathrm{NA}\end{array}$ & $86 \%$ & 0.63 & 0.40 \\
\hline 2 & $\begin{array}{c}\mathrm{GC}_{\text {ratio }} \leq 1.15=\mathrm{A} \\
1.15<\mathrm{GC}_{\text {ratio }} \leq 1.2=\mathrm{PA} \\
1.2<\mathrm{GC}_{\text {ratio }} \leq 1.25=\mathrm{PNA} \\
\mathrm{GC}_{\text {ratio }}>1.25=\mathrm{NA}\end{array}$ & $88 \%$ & 0.81 & 0.66 \\
\hline 3 & $\begin{array}{c}\mathrm{GC}_{\text {ratio }} \leq 1.2=\mathrm{A} \\
1.2<\mathrm{GC}_{\text {ratio }} \leq 1.3=\mathrm{PA} \\
1.3<\mathrm{GC}_{\text {ratio }} \leq 1.35= \\
\mathrm{PNA} \\
\mathrm{GC}_{\text {ratio }}>1.35=\mathrm{NA}\end{array}$ & $86 \%$ & 0.62 & 0.39 \\
\hline 4 & $\begin{array}{c}\mathrm{GC}_{\text {ratio }} \leq 1.2=\mathrm{A} \\
1.2<\mathrm{GC}_{\text {ratio }} \leq 1.35=\mathrm{PA} \\
1.35<\mathrm{GC}_{\text {ratio }} \leq 1.45= \\
\mathrm{PNA} \\
\mathrm{GC}_{\text {ratio }}>1.45=\mathrm{NA}\end{array}$ & $86 \%$ & 0.74 & 0.55 \\
\hline
\end{tabular}



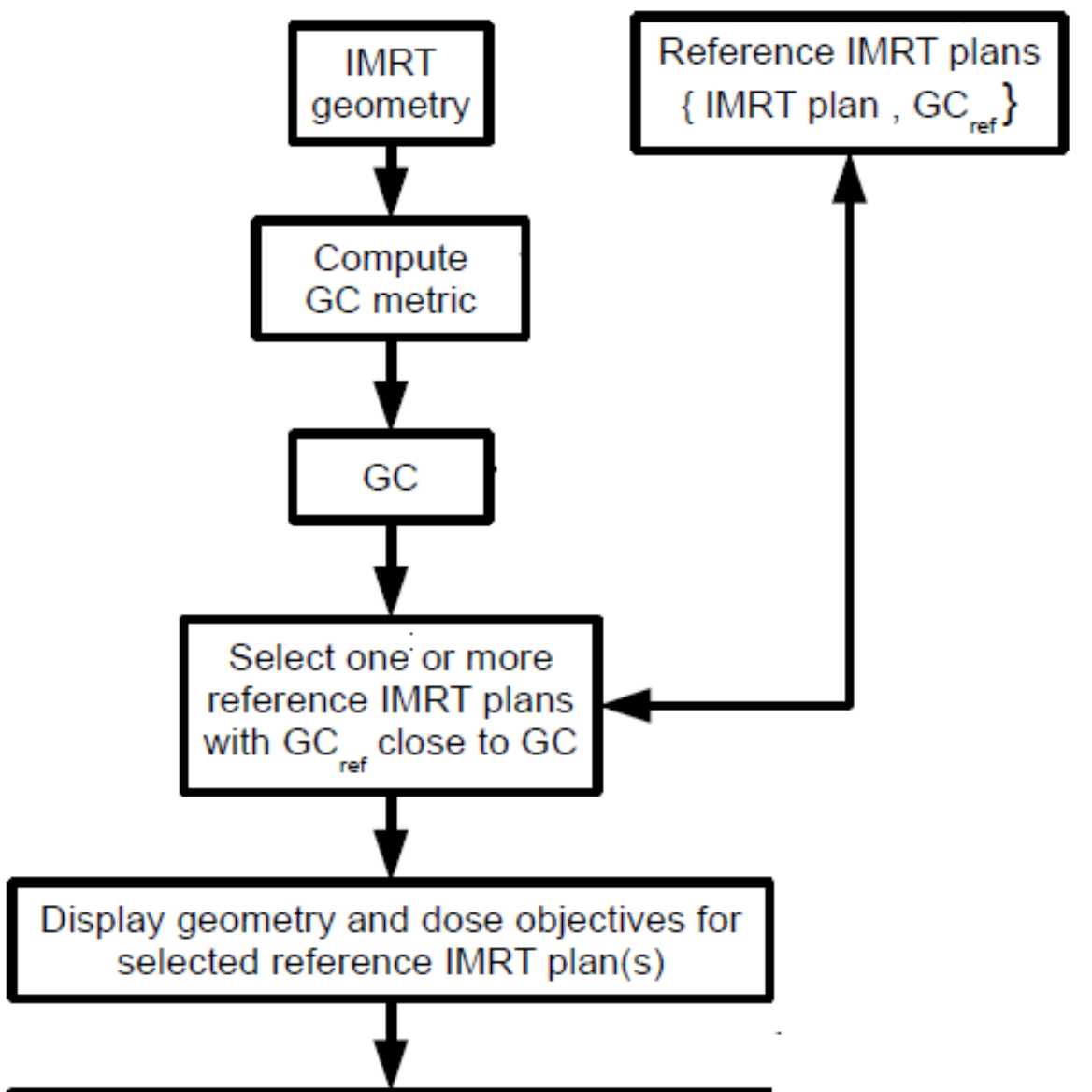

User chooses a best reference plan

Figure 4: An illustration of the process of selecting a good reference plan from a database containing several plans 
Also it is to be noted that the scheme \# 2, which was found to be suitable for interpretation, might not be suitable if a different reference plan is used for prediction. For instance, if Case 3 is used as a reference plan, scheme \# 2 results in an agreement of 340 about $73 \%$ with the obtained results, whereas scheme \# 1 results in an agreement of about $83 \%$. Hence it is important to select an appropriate scheme for interpretation based on the reference plan to make accurate predictions. To select an appropriate scheme, the average $\mathrm{GC}_{\text {ratio }}$ value of a given reference plan can be compared against that of a master reference plan, whose interpretation scheme has been established. Based on the outcome of this

345 comparison, an appropriate interpretation scheme can be picked from a library of schemes for the given reference plan. A unique advantage of the proposed method is that the beam angle configuration between a reference plan and a given plan need not be the same to make predictions. This is because, the computation of geometric complexity directly accounts for the beam configurations.

350

Typically the computation of $\mathrm{GC}_{\text {ratio }}$ takes a few seconds even for the cases involving numerous ROIs. Since, it takes several optimization loops before reaching an acceptable plan in complicated cases, knowing the probable results before invoking optimization can be of very useful. In several different ways a user can respond after knowing the 355 achievability of objectives. For instance, the clinical objectives with lower achievability can be initialized with higher importance weights to ensure they are most likely achieved after optimization, thereby avoiding backtracking steps. This will save a lot of time spent in tweaking the parameters and also reduce the inter-user variability in plan quality. Recently, some researchers have proposed a method for a fully automated solutions for 
360 IMRT optimization [14, 15], which has been implemented in commercial TPS [16-19]. Our approach of predicting the achievability of clinical objectives can also be used in conjunction with the existing automatic approach [16-19] for initializing the objective parameter settings before starting the optimization loops, which can reduce the overall time spent during optimization.

\section{CONCLUSION}

We have proposed a method to predict the achievability of clinical objectives in IMRT planning. Basically our method allows benchmarking a given plan against a high quality reference plan through the use of geometric complexity metric. The study demonstrates

370 the feasibility of using the proposed method for predicting the achievability of clinical objectives with reasonable accuracy. Though we have demonstrated the feasibility for head and neck anatomy, this method should be applicable for other anatomic sites such as brain, thorax, abdomen and pelvis. Also, the proposed method can be directly applied to VMAT scenarios.

375

CONFLICT OF INTEREST

NO CONFLICT OF INTEREST 


\section{REFERENCES:}

1. Purdy. Intensity-modulated radiotherapy: current status and issues of interest. Int $\mathbf{J}$ Radiat Oncol. Biol. Phys. 2001; 51:880-914.

2. Nelms BE, Robinson G, Markham J, Velasco K, Boyd S, Narayan S, Wheeler J, Sobczak ML. Variation in external beam treatment plan quality: An inter-institutional study of planners and planning systems. Practical radiation oncology. 2012; 2:296305.

3. Xing L, Li J, Donaldson S, Le Q, Boyer A. Optimization of importance factors in inverse planning. Med Phys. 1999; 44:2525-36.

4. Zhang X, Wang X, Dong L, Liu H, Mohan R. A sensitivity-guided algorithm for automated determination of IMRT objective function parameters. Med Phys. 2006; $33: 2935-44$.

395 5. Wu and Y. Zhu. An optimization method for importance factors and beam weights based on genetic algorithms for radiotherapy treatment planning. Phys. Med. Biol. $2001 ; 46: 1085-1099$.

6. L. Kessler, D. L. Mcshan, M. A. Epelman, K. A. Vineberg, A. Eisbruch, T. S. Lawrence, and B. A. Fraass.Costlets. A generalized approach to cost functions for automated optimization of IMRT treatment plans. Optimization and Engineering. $2005 ; 6: 421-448$.

7. Stieler F, Yan H, Lohr F, Wenz F, Yin FF. Development of a neuro-fuzzy technique for automated parameter optimization of inverse treatment planning. Radiat Oncol. $2009 ; 4: 39$. 
405 8. Vaitheeswaran R, Narayanan VS, Bhangle J, Nirhali A. TU-A-BRA-05: An Algorithm for Automated Determination of IMRT Objective Function Parameters. Med Phys. 2010; 37:3369.

9. Xhaferllari I, Wong E, Bzdusek K, Lock M, Chen J. Automated IMRT planning with regional optimization using planning scripts. J Appl Clin Med Phys. 2013; 14:17641091.

10. Kazhdan M, Simani P, McNutt T, et al. A shape relationship descriptor for radiation therapy planning. Med Image Comput Comput Assist Interv. 2009; 12:100-08.

11. Wu B, Ricchetti F, Sanguineti G, et al. Patient geometry-driven information retrieval for IMRT treatment plan quality control. Med Phys. 2009; 36:5497-505.

415 12. Wu B, Ricchetti F, Sanguineti G, et al. Data-driven approach to generating achievable dose-volume histogram objectives in intensity-modulated radiotherapy planning. Int $\mathbf{J}$ Radiat Oncol Biol Phys. 2011; 79:1241-47.

13. Wu B, Pang D, Simari P, Taylor R, Sanguineti G, McNutt T. Using overlap volume histogram and IMRT plan data to guide and automate VMAT planning: a head-and420 neck case study. Med Phys. 2013: 40: 021714.

14. Ranganathan V, Kumar P, Bzdusek K, Das JM. SU-G-BRC-01: A Data-Driven Pre-Optimization Method for Prediction of Achievability of Clinical Objectives in IMRT. Med Phys. 2016: 43: 3627

15. Ranganathan, Vaitheeswaran, Prashant Kumar, and Karl Antonin Bzdusek. "Pre425 optimization method for quick prediction of achievability of clinical goals in intensity modulated radiation therapy." U.S. Patent Application No. 16/076,721. 
16. Kumar, Prashant, Karl Antonin Bzdusek, Vaitheeswaran Ranganathan, Matthew Palmer, and Michael Kantor. "Automatic optimal IMRT/VMAT treatment plan generation." U.S. Patent 9,943,702, issued April 17, 2018.

430 17. Kumar, Prashant, Karl Antonin Bzdusek, Vaitheeswaran Ranganathan, Matthew Palmer, and Michael Kantor. "Automatic plan optimization for changing patient anatomy in the presence of mapped delivered dose from delivered fractions." U.S. Patent 10,512,792, issued December 24, 2019.

18. Hazell I, Bzdusek K, Kumar P, Hansen CR, Bertelsen A, Eriksen JG, Johansen J,

435 Brink C. Automatic planning of head and neck treatment plans. J Appl Clin Med Phys. 2016: 17: 272-282.

19. Perumal, B., Sundaresan, H.E., Ranganathan, V., Ramar, N., Anto, G.J. and Meher, S.R., 2019. Evaluation of plan quality improvements in PlanIQ-guided Autoplanning. Reports of Practical Oncology and Radiotherapy, 24(6), pp.533-543.

440 